\title{
Renal Pelvis Adenocarcinoma
}

National Cancer Institute

\section{Source}

National Cancer Institute. Renal Pelvis Adenocarcinoma. NCI Thesaurus. Code C6143.

Adenocarcinoma that affects the renal pelvis. 